\title{
How to Improve Rebound Attacks
}

\author{
María Naya-Plasencia* \\ FHNW, Windisch, Switzerland
}

\begin{abstract}
Rebound attacks are a state-of-the-art analysis method for hash functions. These cryptanalysis methods are based on a well chosen differential path and have been applied to several hash functions from the SHA-3 competition, providing the best known analysis in these cases. In this paper we study rebound attacks in detail and find for a large number of cases that the complexities of existing attacks can be improved.

This is done by identifying problems that optimally adapt to the cryptanalytic situation, and by using better algorithms to find solutions for the differential path. Our improvements affect one particular operation that appears in most rebound attacks and which is often the bottleneck of the attacks. This operation, which varies depending on the attack, can be roughly described as merging large lists. As a result, we introduce new general purpose algorithms for enabling further rebound analysis to be as performant as possible. We illustrate our new algorithms on real hash functions.
\end{abstract}

Keywords: hash functions, SHA-3 competition, rebound attacks, algorithms.

\section{Introduction}

The rebound attack is a recent technique introduced in 13 by Mendel et al. It was conceived to analyze AES-like hash functions (like Grøstl [7] in [14816], Echo [2] in [14818, Whirlpool [1] in [11]). A rebound attack is composed of two parts: the inbound phase and the outbound phase. The aim of the inbound phase is to find, at a low cost, a large number of pairs of values that satisfy a part of a differential path that would be very expensive to satisfy in a probabilistic way. The outbound phase then uses these values to perform an attack.

This technique has been applied to other algorithms with inner permutations which are not AES-like; for instance it has been applied to JH 21 (reduced to 22 rounds) in [17] and Luffa [4] (reduced to 7 rounds) in [10; both of those hash functions use Sboxes of size $4 \times 4$ and have a linear part in which the mixing is done in a very different way than in the AES. The hash function LANE 9, which includes several AES states, each treated by the AES round transformation, and a different transformation for mixing these states has also been analysed in 1222 using rebound attacks.

\footnotetext{
* Supported by the National Competence Center in Research on Mobile Information and Communication Systems (NCCR-MICS), a center of the Swiss National Science Foundation under grant number 5005-67322.
} 
In these cryptanalysis results, the rebound attack technique needs to be refined and adapted to each case, but all of them follow the same scheme: first find a differential path, then find solutions verifying this differential path. This paper focuses on optimizing the latter part. In all the previously mentioned cryptanalysis, that part involves enumerating, from a very large set of possible candidates represented as a cross product of lists, all those that verify a given relation. We call this operation "merging" the lists. The merging problem can be described more formally as follows.

Merging problem with respect to $t$ : Let $t$ be a Boolean function taking $N k$-bit words as input, i.e. $t:\left(\{0,1\}^{k}\right)^{N} \rightarrow\{0,1\}$. Let $L_{1}, \ldots, L_{N}$ be $N$ given lists of $k$-bit words drawn uniformly and independently at random from $\{0,1\}^{k}$. We assume that the probability over all $N$-tuples $X$ in $L_{1} \times \ldots \times L_{N}$ that $t(X)=1$ is $P_{t}$. For any given function $t$ and any given $N$-tuple of lists $\left(L_{1}, \ldots, L_{N}\right)$ the merging problem consists in finding the list $\mathcal{L}_{\text {sol }}$ of all $X \in L_{1} \times \ldots \times L_{N}$ satisfying $t(X)=1$. We call this operation merging the lists $L_{1}, \ldots, L_{N}$ to obtain $\mathcal{L}_{\text {sol }}$.

It is assumed that the image of a given input under $t$ can be easily computed. In the following, the size of a list $L$ is denoted by $|L|$. A brute force method for solving this problem therefore consists in enumerating all the $\left|L_{1}\right| \times \ldots \times\left|L_{N}\right|$ inputs, in computing $t$ on all of them and in keeping the ones verifying $t=1$. Note that, in the lack of any additional information on $t$, it is theoretically impossible to do better. However, in practice, the function $t$ often has a set of properties which can be exploited to optimize this approach. We aim at reducing the number of candidates which have to be examined, in some cases by a preliminary sieving similar to the one used in [5]. This paper presents such optimization techniques, that, when applied to most of the rebound attacks published on the SHA-3 candidates, yield significant improvements in the overall time and/or memory complexities of the attack, as shown on Table 1. In this table we can see that we have considered the best existing attacks against four hash functions and the best rebound attack on a fifth (two of them are finalists and two are second-round candidates of the SHA-3 competition), where by best attack we denote the one on the highest number of rounds. We have been able to improve their complexities by scrutinizing the original attack and finding a more efficient algorithm for obtaining the solutions for the differential path. Most of the time the improvement relies on a better merging of the lists, and sometimes it is due to the use of more adequate conditions in the general algorithm. Let us recall here that the aim is to find all the $N$-tuples that verify $t=1$ for a complex function $t$, which is significantly different from finding just one (or few) of them for a linear $t$ such as in 201963. As in the previous rebound analysis, we will work throughout the paper with average values in the probabilistic cases.

In Section 2, we define Problem 1 that corresponds to functions $t$ with a particular form, and we propose three generic algorithms to solve it. These 3 algorithms have different optimal scenarios. Some examples of applications are given. In Section 3 we define Problem 2 and propose the stop-in-the-middle algorithms for solving it. We also present two concrete algorithms in this family 
Table 1. Improvements on best known attacks. The highlighted values are the improved complexities. For Luffa we consider the best known rebound attack where the complexities presented in the second row have already been obtained in [10] by a dedicated algorithm similar to our general approach.

\begin{tabular}{|c|c|c|c|c|c|c|c|c|}
\hline \multirow{2}{*}{ Hash function } & \multirow{2}{*}{$\begin{array}{l}\text { SHA3 } \\
\text { Round }\end{array}$} & \multirow{2}{*}{ Best Known Analysis } & \multirow{2}{*}{$\begin{array}{r}\text { Rounds } \\
/ \text { total }\end{array}$} & \multicolumn{3}{|c|}{ Previous } & \multicolumn{2}{|c|}{ This paper } \\
\hline & & & & Time & Memory & Ref. & Time & Memory \\
\hline $\begin{array}{l}\mathrm{JH} \\
\mathrm{JH}\end{array}$ & Final & $\begin{array}{r}\text { semi-free-start coll. } \\
\text { semi-free-start near coll. }\end{array}$ & $\begin{array}{l}16 / 42 \\
22 / 42\end{array}$ & $\begin{array}{l}2^{190} \\
2^{168}\end{array}$ & $\begin{array}{r}2^{104} \\
2^{143.70}\end{array}$ & $\begin{array}{l}17 \\
17 \\
\end{array}$ & $\begin{array}{l}2^{97} \\
2^{96}\end{array}$ & $\begin{array}{l}2^{97} \\
2^{96}\end{array}$ \\
\hline $\begin{array}{l}\text { Grøstl-256 } \\
\text { Grøstl-256 } \\
\text { Grøstl-512 } \\
\end{array}$ & Final $^{*}$ & $\begin{array}{r}\text { (compr. function property) } \\
\text { (internal permutation dist.) } \\
\text { (compr. function property) }\end{array}$ & $\begin{array}{l}10 / 10 \\
10 / 10 \\
11 / 14\end{array}$ & $\begin{array}{l}2^{192} \\
2^{192} \\
2^{640}\end{array}$ & $\begin{array}{l}2^{64} \\
2^{64} \\
2^{64}\end{array}$ & $\begin{array}{l}16 \\
16 \\
16 \\
\end{array}$ & $\begin{array}{l}2^{182} \\
2^{175} \\
2^{630} \\
\end{array}$ & $\begin{array}{l}2^{64} \\
2^{64} \\
2^{64} \\
\end{array}$ \\
\hline ECHO-256 & $2^{\text {nd }}$ & internal permutation dist. & $8 / 8 \mid$ & $2^{182}$ & $2^{37}$ & 18 & $2^{151}$ & $2^{67}$ \\
\hline Luffa & $2^{\text {nd }}$ & semi-free-start coll. & $7 / 8$ & $2^{132}$ & $2^{68.8}$ & 10 & $\begin{array}{l}2^{112.9} \\
\left(2^{104}\right)\end{array}$ & $\begin{array}{r}2^{68.8} \\
\left(2^{102}\right) \\
\end{array}$ \\
\hline $\begin{array}{l}\text { LANE- } 256 \\
\text { LANE-512 }\end{array}$ & $1^{s t}$ & $\begin{array}{l}\text { semi-free-start coll. } \\
\text { semi-free-start coll. }\end{array}$ & $\begin{array}{l}6+3 / 6+3 \\
8+4 / 8+4\end{array}$ & $\begin{array}{r}2^{96} \\
2^{224}\end{array}$ & $\begin{array}{r}2^{88} \\
2^{128}\end{array}$ & \begin{tabular}{|l|}
12 \\
12 \\
12
\end{tabular} & $\begin{array}{r}\mathbf{2}^{\mathbf{8 0}} \\
2^{224}\end{array}$ & $\begin{array}{l}2^{66} \\
2^{66}\end{array}$ \\
\hline
\end{tabular}

* The Grøstl analysis does not apply after the final round tweak.

applied to the scenarios of ECHO and LANE. In Section 4 we show briefly how applying these algorithms combined with an appropriate definition and decomposition of the problem in each case, allows us to improve the complexities of the best known rebound attacks on 5 SHA-3 candidates. Besides the results in Table 1, the main interest of this paper is to present a general framework for improving rebound attacks. We introduce several new algorithms that considerably improve the overall effectiveness when the attack needs to merge large lists. We provide a formal definition of the field of application of those algorithms, and describe them as a set of constraints on $t$, in hope that designers of rebound attacks will be able to easily identify scenarios where one of these algorithms, or variants, may be applied. This was motivated by our own research path, when we realized that a generalization of the techniques leveraged in specific cases allowed us to find similar improvements in almost all of the rebound attacks that we have studied so far.

\section{When $t$ is Group-Wise}

In some cases we can considerably reduce the complexity of the merging problem by redefining it into a more concrete one. We consider here a very common case that will appear in many rebound scenarios, as we will later show with the examples. This case corresponds to a function $t$ that can be decomposed in smaller functions. After introducing the general problem, we will illustrate it with an example. Though we preferred to state the problem in full generality for any possible $N$, in the concrete rebound examples that we studied, the number of lists $N$ was either 2, 4 or 6 . Also, the elements of each list can be decomposed in sets of small size $s$, where $s$ is typically the size of the involved Sbox; and $z$ is the number of such sets involved 1 in the function $t$.

\footnotetext{
${ }^{1}$ Sometimes, elements are only partially involved in $t$.
} 
Problem 1: Let $L_{1}, \ldots, L_{N}$ be $N$ lists of size $2^{l_{1}}, \ldots, 2^{l_{N}}$ respectively, where the elements are drawn uniformly and independently at random from $\{0,1\}^{k}$.

Let $t$ be a Boolean function, $t:\left(\{0,1\}^{k}\right)^{N} \rightarrow\{0,1\}$ for which there exists $N^{\prime}<N$, an integer $z$ and some triples of functions $t_{j}:\{0,1\}^{2 s} \rightarrow\{0,1\}$, $f_{j}:\left(\{0,1\}^{k}\right)^{N^{\prime}} \rightarrow\{0,1\}^{s}$ and $f_{j}^{\prime}:\left(\{0,1\}^{k}\right)^{\left(N-N^{\prime}\right)} \rightarrow\{0,1\}^{s}$ for $j=1, \ldots, z$ such that, $\forall\left(\boldsymbol{x}_{1}, \ldots, \boldsymbol{x}_{N}\right) \in L_{1} \times \ldots \times L_{N}$ :

$$
\begin{gathered}
\forall j=1, \ldots, z, \\
t\left(\boldsymbol{x}_{\mathbf{1}}, \ldots, \boldsymbol{x}_{\boldsymbol{N}}\right)=1 \quad \Leftrightarrow \quad\left\{\begin{array}{l}
t_{j}\left(v_{j}, v_{j}^{\prime}\right)=1 \\
\text { with } v_{j}=f_{j}\left(\boldsymbol{x}_{\mathbf{1}}, \ldots, \boldsymbol{x}_{\boldsymbol{N}^{\prime}}\right) \\
\text { and } v_{j}^{\prime}=f_{j}^{\prime}\left(\boldsymbol{x}_{\boldsymbol{N}^{\prime}+\mathbf{1}}, \ldots, \boldsymbol{x}_{\boldsymbol{N}}\right)
\end{array}\right.
\end{gathered}
$$

Let $P_{t}$ be the probability that $t=1$ for a random input.

Problem 1 consists in merging these $N$ lists to obtain the set $\mathcal{L}_{\text {sol }}$, of size $P_{t} 2^{\sum_{i=1}^{N} l_{i}}$, of all $N$-tuples of $\left(L_{1} \times \ldots \times L_{N}\right)$ verifying $t=1$.

Reduction from $N$ to 2: For any $N \geq 2$ Problem 1 can be reduced to an equivalent and simplified problem with $N=2$, i.e. merging two lists $L_{A}$ and $L_{B}$, which consist of elements in $\left(\{0,1\}^{s}\right)^{z}$ corresponding to $\boldsymbol{x}_{\boldsymbol{A}}=\boldsymbol{v}=\left(v_{1}, \ldots, v_{z}\right)$ and $\boldsymbol{x}_{\boldsymbol{B}}=\boldsymbol{v}^{\prime}=\left(v_{1}^{\prime}, \ldots, v_{z}^{\prime}\right)$, with respect to the function $\boldsymbol{x}_{\boldsymbol{A}}, \boldsymbol{x}_{\boldsymbol{B}} \mapsto \Pi_{j=1}^{z} t_{j}\left(v_{j}, v_{j}^{\prime}\right)$. The reduction is performed as follows:

1. Build a table $T_{A}^{*}$ of size $2^{\sum_{i=1}^{N^{\prime}} l_{i}}$ storing each element $\boldsymbol{e}_{\boldsymbol{A}}=\left(\boldsymbol{x}_{\mathbf{1}}, \ldots, \boldsymbol{x}_{\boldsymbol{N}^{\prime}}\right)$ of $L_{1} \times \ldots \times L_{N^{\prime}}$, indexed 2 by the value of $\left(f_{1}\left(\boldsymbol{e}_{\boldsymbol{A}}\right), \ldots, f_{z}\left(\boldsymbol{e}_{\boldsymbol{A}}\right)\right)$, i.e. $\left(v_{1}, \ldots, v_{z}\right)$. Store the corresponding $\left(v_{1}, \ldots, v_{z}\right)$ in a list $L_{A}$. Note that several $\boldsymbol{e}_{\boldsymbol{A}}$ may lead to the same value of $\left(v_{1}, \ldots, v_{z}\right)$.

2. Build a similar table $T_{B}^{*}$ of size $2^{\sum_{i=N^{\prime}+1}^{N} l_{i}}$ storing each element $\boldsymbol{e}_{\boldsymbol{B}}=$ $\left(\boldsymbol{x}_{\boldsymbol{N}^{\prime}+\mathbf{1}}, \ldots, \boldsymbol{x}_{\boldsymbol{N}}\right)$ of $L_{N^{\prime}+1} \times \ldots \times L_{N}$, indexed by $\left(f_{1}\left(\boldsymbol{e}_{\boldsymbol{B}}\right), \ldots, f_{z}\left(\boldsymbol{e}_{\boldsymbol{B}}\right)\right)$, i.e. $\left(v_{1}^{\prime}, \ldots, v_{z}^{\prime}\right)$. Store $\left(v_{1}^{\prime}, \ldots, v_{z}^{\prime}\right)$ in a list $L_{B}$.

3. Merge $L_{A}$ and $L_{B}$ with respect to $\Pi_{j=1}^{z} t_{j}$ and obtain $\mathcal{L}_{\text {sol }}$.

4. Build $\mathcal{L}_{\text {sol }}^{*}$ by iterating over each pair $\left(\left(v_{1}, \ldots, v_{z}\right),\left(v_{1}^{\prime}, \ldots, v_{z}^{\prime}\right)\right)$ of $\mathcal{L}_{\text {sol }}$, and adding the set of all $\left(\boldsymbol{x}_{\mathbf{1}}, \ldots, \boldsymbol{x}_{\boldsymbol{N}^{\prime}}, \boldsymbol{x}_{\boldsymbol{N}+\mathbf{1}}, \ldots, \boldsymbol{x}_{\boldsymbol{N}}\right) \in T_{A}^{*}\left[\left(v_{1}, \ldots, v_{z}\right)\right] \times$ $T_{B}^{*}\left[\left(v_{1}^{\prime}, \ldots, v_{z}^{\prime}\right)\right] . \mathcal{L}_{\text {sol }}^{*}$ is the solution to the original problem.

Let $2^{T_{\text {merge }}}, 2^{M_{\text {merge }}}$ be the time and memory complexities of step 3 . The total time complexity of solving Problem 1 is $\mathcal{O}\left(s z 2^{\sum_{i=1}^{N^{\prime}} l_{i}}+s z 2^{\sum_{i=N^{\prime}+1}^{N} l_{i}}+2^{T_{\text {merge }}}+\right.$ $P_{t} 2^{\sum_{i=1}^{N} l_{i}}$ ) where the last term comes from the fact that only the $N$-tuples satisfying $t=1$ are examined at step 4 because of the sieve applied at step 3 . The proportion of such tuples is then $P_{t}$. The memory complexity ${ }^{3}$ is $\mathcal{O}((z s+$ $\left.N^{\prime} k\right) 2^{\sum_{i=1}^{N^{\prime}} l_{i}}+\left(z s+\left(N-N^{\prime}\right) k\right) 2^{\sum_{i=N^{\prime}+1}^{N} l_{i}}+2^{M_{\text {merge }}}+P_{t} 2^{\sum_{i=1}^{N} l_{i}}$, where the last term appears only when the solutions must be stored.

${ }^{2}$ Here and in the following sections we can use standard hash tables for storage and lookup in constant time, since the keys are integers.

3 The first two terms, corresponding to the storage of $T_{A}^{*}$ and $T_{B}^{*}$ could be avoided if they were the bottleneck by slightly increasing the time complexity by a factor of 2 . 
Using the brute force approach, $2^{T_{\text {merge }}}$ would be $2^{l_{A}+l_{B}}$ where $2^{l_{A}}$ (respectively $\left.2^{l_{B}}\right)$ denotes the size of $L_{A}\left(L_{B}\right)$, and $2^{M_{\text {merge }}}$ would be negligible. We present in the following sections some algorithms for solving Problem 1 considering $N=2$ with $L_{A}$ and $L_{B}$, that provide better complexities than the brute force approach. Note that the roles of $L_{A}$ and $L_{B}$ are assigned by choice to obtain the best overall complexity. Those algorithms can be applied for obtaining a smaller $2^{T_{\text {merge }}}$ when $N>2$.

\subsection{Basic Algorithm for Solving Problem 1: Instant Matching}

As $s$ is typically very small we can enumerate the solutions $\left(v_{j}, v_{j}^{\prime}\right)$ of $t_{j}\left(v_{j}, v_{j}^{\prime}\right)=$ 1 and store them in tables $T_{j}$ of size $\leq 2^{2 s}$, indexed by $v_{j}^{\prime}$. This costs $\mathcal{O}\left(z \cdot 2^{2 s}\right)$ in time and memory. We propose in Fig. 1 a first algorithm for solving Problem 1, which has lower complexity than the brute-force approach. Although being the simplest algorithm presented in this paper, it has not been applied in critical steps of some of the previously mentioned attacks, though it could yield significant improvements.

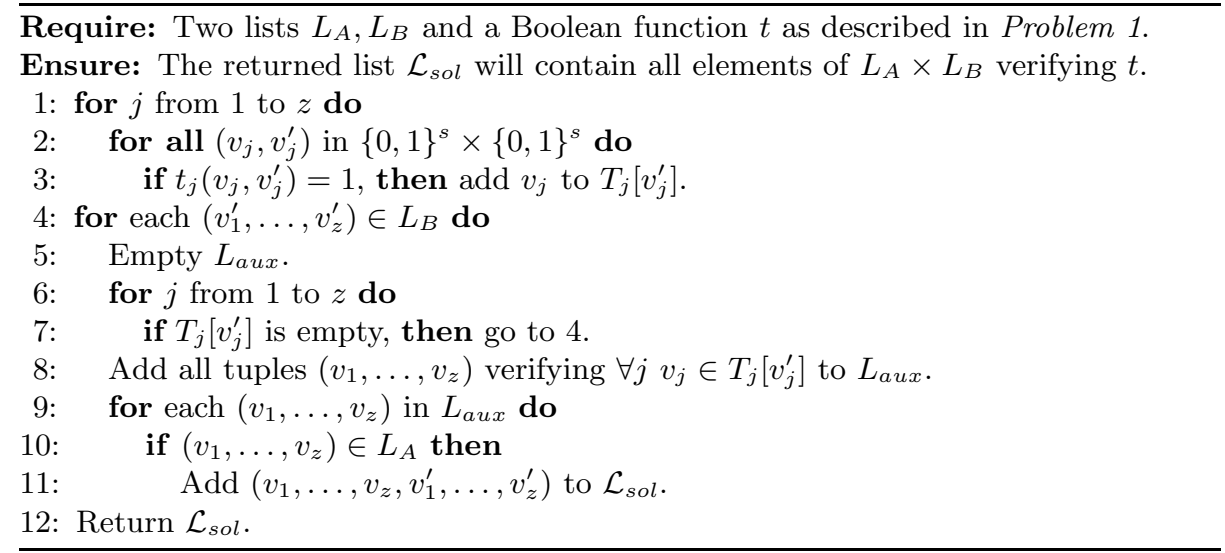

Fig. 1. Instant matching algorithm

Let $2^{-p_{j}}$ be the probability over all pairs $\left(v_{j}, v_{j}^{\prime}\right)$ that $t_{j}\left(v_{j}, v_{j}^{\prime}\right)=1$. The relationship between $t$ and the $\left(t_{j}\right)_{1 \leq j \leq z}$ implies that $\sum_{j=1}^{z} p_{j}=-\log _{2}\left(P_{t}\right)$ where $P_{t}$ is the probability that $t=1$.

Let us determine the average size of $L_{a u x}$. The average size of $T_{j}\left[v_{j}^{\prime}\right]$ over all $v_{j}^{\prime}$ is $2^{s-p_{j}}$. Then the average size of $L_{a u x}$ is $2^{z s-\sum_{j=1}^{z} p_{j}}=P_{t} 2^{z s}$. It follows that the time complexity of the algorithm is $\mathcal{O}\left(z 2^{s}+z P_{t} 2^{l_{B}+z s}\right)$ and is proportional to the product of the size of $L_{B}$ by the average size of $L_{\text {aux }}$. The memory complexity is $\mathcal{O}\left(z 2^{s}+2^{l_{A}}+2^{l_{B}}+P_{t} 2^{l_{A}+l_{B}}\right)$. In some cases, the last term can disappear, namely if we do not need to store the list $\mathcal{L}_{\text {sol }}$, but just use each solution as soon as it is obtained. The same way, the list $L_{B}$ does not need to be stored, if it can be given on the fly.

4 The cost of building and storing the lists $T_{j}\left[v_{j}^{\prime}\right]$ is negligible. 
We now describe a concrete example of application of the instant-matching algorithm in a case included in a particular rebound attack, improving its complexity. In the extended version of this paper [15] two more examples are provided in Appendix A, where it clearly appears that identifying and isolating the most appropriate problem (or problems) to solve is of major importance. These two last examples might help also to understand the role of $f_{j}$ and $f_{j}^{\prime}$.

Example 1: Application of the Instant Matching Algorithm. We use here a case presented in the analysis of $\mathrm{JH}$ [17] which is the attack on 8 rounds using one inbound when the dimension of a block of bits denoted by $d$ is 4 . Here we improve step 3 of the attack, which is also the bottleneck in time complexity. Two lists are given, $L_{A}$ and $L_{B}$ of size $2^{24.18}$ elements each. The aim of step 3 is to merge those lists, i.e. find all pairs $\left(\boldsymbol{v}, \boldsymbol{v}^{\prime}\right) \in L_{A} \times \mathrm{Ł}_{B}$ verifying 10 conditions on groups of $s=4$ bits of $\left(\boldsymbol{v}, \boldsymbol{v}^{\prime}\right)$.

In [17] this is solved by exhaustive search, i.e. all possible pairs are examined and only the ones that verify the 10 conditions are kept, which has cost $2^{48.36}$. We can improve this complexity by applying the instant-matching algorithm: first, we notice that 6 out of these 10 conditions can be written as

$$
t_{j}\left(v_{j}, v_{j}^{\prime}\right)=1, \forall j \in\{1, \ldots, 6\},
$$

where variables $v_{j}$ and $v_{j}^{\prime}$ represent groups of differences of 4 bits. The functions $t_{j}$ return 1 when the linear function of $\mathrm{JH}, L$, applied to $v_{j}$ and $v_{j}^{\prime}$ produces 4 bits out of 8 without difference in the wanted positions. Those functions $t_{j}$ can be computed directly by using a precomputed table of size $2^{8}$.

This is an instance of Problem 1 with the parameters: $z=6$ (corresponding to the number of relations $\left.t_{1}, \ldots, t_{6}\right)$, and $p_{j}=3.91 \forall j$. Hence $P_{t} 2^{z s}=2^{0.09 \cdot 6}=$ $2^{0.54} \simeq 1.45$. The instant-matching algorithm allows us to find all pairs satisfying these 6 conditions with a complexity of $2^{27.8}$ in time and no additional memory. We then obtain $2^{24.9}$ pairs of elements that pass the first 6 conditions. To complete step 3 of the attack, we evaluate the 4 remaining conditions for each pair, for a global complexity of $2^{24.9}$.

To summarize, we were able to resolve step 3 of the attack with a time complexity of about $2^{27.8}$, improving significantly the complexity of $2^{48.36}$ given in [17].

\subsection{Solving Problem 1 When $P_{t} 2^{z s}>2^{l_{A}}$ : Gradual Matching}

In Fig. 2 we present an algorithm for solving Problem 1 that is useful in cases where the average size of $L_{a u x}$ exceeds the size of $L_{A}$, i.e $5 P_{t} 2^{z s}>2^{l_{A}}$. In this case the instant-matching algorithm has a higher complexity than the exhaustive search. This is why here, instead of directly matching the $z$ groups that appear in relation $t$, we will first match the $z^{\prime}<z$ ones, and next, the $z-z^{\prime}$ remaining

${ }^{5}$ When $P_{t} 2^{z s}$ is close to $2^{l}$ this algorithm might also outperform the instant-matching technique. 
ones. We present here how to use one step of the gradual-matching algorithm for solving Problem 1. This algorithm reminds the method used in Example 1 where the problem is first solved with only 6 relations. But the difference is that the remaining $z-z^{\prime}$ relations can also be written in the form needed for Problem 1 and $P_{t} 2^{z s}>2^{l_{A}}$. Let us suppose that we choose $z^{\prime}$ so that $z^{\prime} s<l_{A}$ (the best value for $z^{\prime}$ depends on the situation).

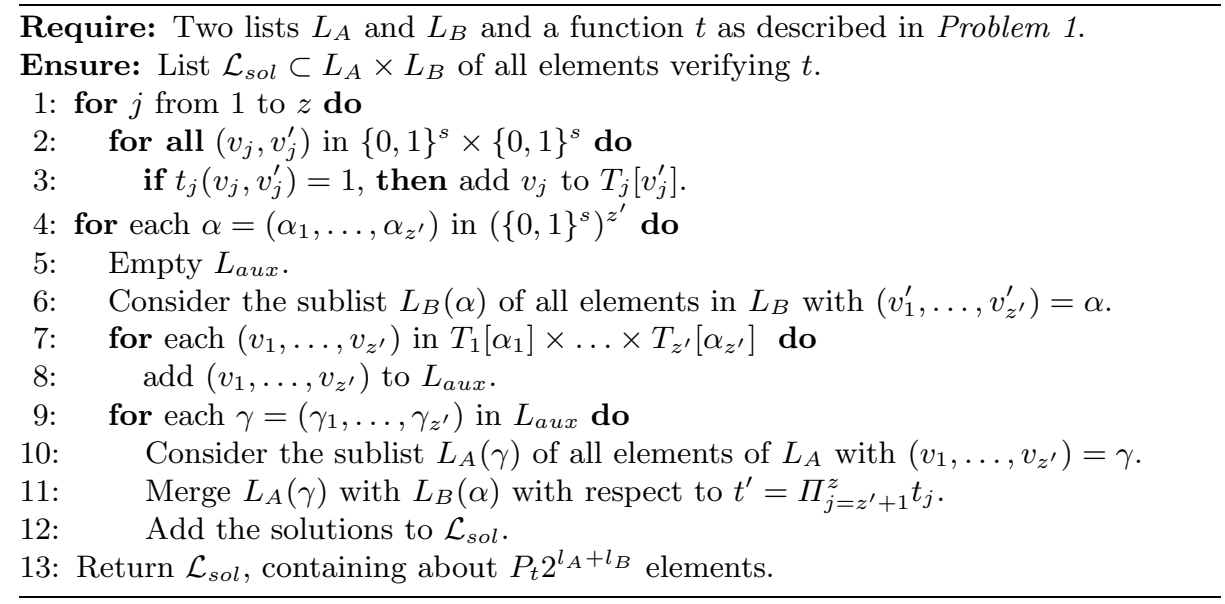

Fig. 2. Gradual matching algorithm

Let $2^{\text {merge }}$ be the time complexity of merging once lists $L_{B}(\alpha)$ and $L_{A}(\gamma)$ as defined in Fig. 2. Since their respective average sizes are $2^{l_{A}-z^{\prime} s}$ and $2^{l_{B}-z^{\prime} s}$ the complexity of the brute force is $2^{l_{A}+l_{B}-2 z^{\prime} s}$. It can be improved by using one of the proposed algorithms from this section but it cannot be smaller than the size of the resulting merged list, i.e. $2^{l_{A}+l_{B}-2 z^{\prime} s-\sum_{j=z^{\prime}+1}^{z} p_{j}}$. Now the average size of $L_{a u x} \sqrt{6}$ is $\mathcal{S}=2^{z^{\prime} s-\sum_{j=1}^{z^{\prime}} p_{j}}$. Then, the time complexity of this algorithm is $\mathcal{O}\left(z 2^{s}+2^{z^{\prime} s}\left(z^{\prime}+\mathcal{S} 2^{\text {merge }}\right)\right)$. It is worth noticing that this complexity corresponds to $z^{\prime} 2^{z^{\prime} s}+2^{l_{A}+l_{B}-\sum_{j=1}^{z^{\prime}} p_{j}}$ when the intermediate lists are merged by the brute force algorithm and to $z^{\prime} 2^{z^{\prime} s}+P_{t} 2^{l_{A}+l_{B}}$ if they are merged by an optimal algorithm. The memory complexity is $\mathcal{O}\left(z 2^{s}+2^{l_{A}}+2^{l_{B}}+\mathcal{S}+P_{t} 2^{l_{A}+l_{B}}\right)$. Again, in some cases, the last term can disappear, if we do not need to store the list $\mathcal{L}_{\text {sol }}$, but just use the solutions on the fly.

\subsection{Time-Memory Trade-Offs When $P_{t} 2^{z s}>2^{l_{A}}$ : Parallel Matching}

The parallel-matching algorithm improves the time complexity of the gradualmatching by a time-memory trade-off and can be applied in the same situations.

${ }^{6}$ Here and in the previous section, there is no need for storing $L_{a u x}$, as each element can be treated as soon as it is obtained, but these auxiliary lists are very useful for describing the complexities. 
It is a generalization of an algorithm proposed in [10. As the gradual-matching algorithm this algorithm first finds elements that verify $t_{j}=1$ for $j \in\left\{1, \ldots, z^{\prime}\right\}$ and then, for each of them, it checks if the remaining $\left(z-z^{\prime}\right)$ relations are also verified. However, in this algorithm, the matching of the $z^{\prime}$ relations is done in parallel for $n$ and $m$ relations, so that $z^{\prime}=m+n$. The motivation of choosing different variables for $n$ and $m$ is showing that there is no need for them to be the same when applying the algorithm. We choose $n$ so that $n<z$, ns $<l_{A}$
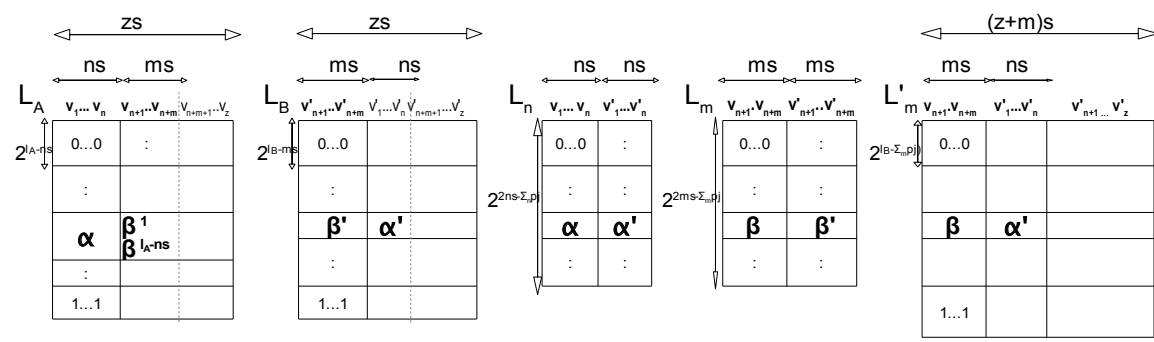

Fig. 3. Representation of the parallel-matching algorithm

and $n s<l_{B}$, and in the same way, we choose $m\left(n+m=z^{\prime} \leq z\right)$. This algorithm will be explained with ordered lists, as it is more graphical and helps the understanding. However, since we can perform it with hash tables indexed by the values we want to have ordered, we do not need to take into account the logarithmic terms for ordering and searching in the final complexity. First we build the lists that we will use and that are represented in Fig. 3 .

- We order the list $L_{A}$ by the first $n$ groups $\left(v_{1}, \ldots, v_{n}\right) . L_{A}$ has $2^{l_{A}-s n}$ elements in average corresponding to a given value of these $n$ groups.

- We order the list $L_{B}$ by the next $m$ groups $\left(v_{n+1}^{\prime}, \ldots, v_{n+m}^{\prime}\right) . L_{B}$ has $2^{l_{B}-s m}$ elements in average corresponding to a given value of these $m$ groups.

- We build the list $L_{n}$ of size $2^{2 n s-\sum_{j=1}^{n} p_{j}}$ formed by all $\left(v_{1}, \ldots, v_{n}, v_{1}^{\prime}, \ldots, v_{n}^{\prime}\right)$ with $v_{j} \in T_{j}\left[v_{j}^{\prime}\right]$ for all $1 \leq j \leq n$. All the elements from this list satisfy $t_{j}\left(v_{j}, v_{j}^{\prime}\right)=1$ for $j \in[1, \ldots, n]$.

- We build the list $L_{m}$ of size $2^{2 m s-\sum_{j=n+1}^{n+m} p_{j}}$ formed by all $\left(v_{n+1}, \ldots, v_{n+m}\right.$, $\left.v_{n+1}^{\prime}, \ldots, v_{n+m}^{\prime}\right)$ with $v_{j} \in T_{j}\left[v_{j}^{\prime}\right]$ for all $(n+1) \leq j \leq(n+m)$. All the elements from this list satisfy $t_{j}\left(v_{j}, v_{j}^{\prime}\right)=1$ for $j \in[n+1, \ldots, n+m]$.

- From $L_{m}$ and $L_{B}$ we build $L_{m}^{\prime}$ as follows: for each $\left(\beta, \beta^{\prime}\right)$ in $L_{m}$, we add to $L_{m}^{\prime}$ all elements $\left(\beta, v_{1}^{\prime}, \ldots, v_{z}^{\prime}\right)$ of $L_{B}$ such that $\left(v_{n+1}^{\prime}, \ldots, v_{n+m}^{\prime}\right)=\beta^{\prime}$ and we store them ordered by the values of $\left(\beta, v_{1}^{\prime}, \ldots, v_{n}^{\prime}\right)$. The average size of $L_{m}^{\prime}$ is $2^{l_{B}+s m-\sum_{j=n+1}^{n+m} p_{j}}$. Then we perform the algorithm given in Fig. 4 .

In total we find the $2^{l_{A}+l_{B}-\sum_{j=1}^{z} p_{j}}$ existing matches, with a complexity of $\mathcal{O}\left(2^{l_{n}}+2^{l_{m}}+2^{l_{A}+l_{B}-\sum_{j=1}^{n+m} p_{j}}+2^{l_{A}+n s-\sum_{j=1}^{n} p_{j}}+2^{l_{B}+m s-\sum_{j=n+1}^{m} p_{j}}\right)$ in time 


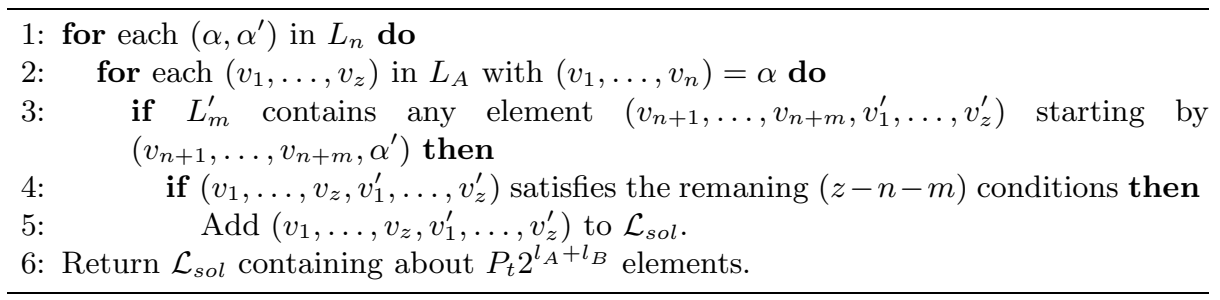

Fig. 4. Parallel matching algorithm

and $\mathcal{O}\left(2^{l_{n}}+2^{l_{m}}+2^{l_{B}}+2^{l_{B}+m s-\sum_{j=n+1}^{m} p_{j}}+2^{l_{A}+l_{B}-\sum_{j=1}^{z} p_{j}}\right)$ in memory, where the last term corresponds to the storage of all solutions, not always needed. In this case, the storage of $L_{A}$ is not necessary.

\subsection{Example 2: Gradual Matching vs Parallel Matching}

We are going to apply both previous algorithms to the analysis of Luffa presented in [10. We are given two lists $L_{A}$ and $L_{B}$ of size $2^{67}$ and $2^{65.6}$. These lists contain elements formed by $z=52$ groups of differences of $s=4$ bits. List $L_{A}$ contains the possible differences for the input of 52 Sboxes. List $L_{B}$ contains the possible differences for the output of the same 52 Sboxes. For the $j$-th Sbox, the probability that one input difference can be associated to one output difference is $2^{-p_{j}}=2^{-1.23}$. The average size of $L_{a u x}$ if we apply the instant-matching algorithm is then $P_{t} 2^{z s}=2^{144.04}$. In this case $t$ can be decomposed in $52 t_{j}$, one per Sbox. So $t_{j}\left(v_{j}, v_{j}^{\prime}\right)=1$ if there exists $x \in\{0,1\}^{s}$ such that

$$
\operatorname{Sbox}(x) \oplus \operatorname{Sbox}\left(x \oplus v_{j}\right)=v_{j}^{\prime} .
$$

The brute force algorithm for solving this problem has complexity of $2^{65.6+67}=$ $2^{132.6}$ in time and of $2^{68.8}$ in memory. If we apply the gradual-matching algorithm with $z^{\prime}=16$ we have $\mathcal{S}=2^{44.32}$, and we obtain the $2^{68.8}$ solutions with a time complexity of $2^{112.9}$ and the same memory as before as no additional memory is needed. If instead we apply the parallel-matching algorithm with $m=n=13$, we can obtain the solutions with a time complexity of $2^{104}$ and a memory complexity of $2^{102}$. Different choices of parameters allow many other time-memory tradeoffs, but we just show here the one that provides the lowest time complexity, and so the highest memory needs, for contrast with the gradual matching algorithm.

\section{Stop-in-the-Middle Algorithms}

In this section we present another case that allows to reduce the complexity of solving the basic problem. It is described in Problem 2. Then, we define the main lines of the stop-in-the-middle algorithms, that we use for solving Problem 2. Next, we present such an algorithm that solves Problem 2 in the scenario of LANE-256. Then a more complex variant of this algorithm is applied to an 
ECHO-256 scenario. We believe that, in particular, this kind of algorithms can be adapted and applied to functions that use several AES (like) states in parallel which are then merged at the end of each round.

In the following, we consider a permutation $F$ from $\{0,1\}^{s k}$ to $\{0,1\}^{s k}$ and we assume that there exists a decomposition function $\phi$ (respectively $\psi$ ) of the input of $F$ (respectively the output) in $k$ elements of $\{0,1\}^{s}$. These two decompositions may be different. Then, instead of the original function $F$ we will now focus on the function $f=\psi \circ F \circ \phi^{-1}$ which is a function over $\left(\{0,1\}^{s}\right)^{k}$ (see Fig. 51). In the following $(u, w)$ denotes the word corresponding to the concatenation of the vectors $u$ and $w$.

Problem 2: Let $z_{A}$ and $z_{B}$ be two integers less than or equal to $k$. Let $L_{A}$ be a list of elements in $\left(\{0,1\}^{s}\right)^{z_{A}}$ and $L_{B}$ be a list of elements on $\left(\{0,1\}^{s}\right)^{z_{B}}$. The Problem 2 consists on finding all triples $(a, b, c)$ with $a \in L_{A}, b \in L_{B}$ and $c \in L_{C}=\left(\{0,1\}^{s}\right)^{k}$ such that

$$
f(c) \oplus f\left(c \oplus\left(a, 0^{s\left(k-z_{A}\right)}\right)\right)=\left(b, 0^{s\left(k-z_{B}\right)}\right),
$$

where there exists the function $F_{1}:\left(\{0,1\}^{s}\right)^{k} \rightarrow\left(\{0,1\}^{s}\right)^{k}$ and some permutations of $\{0,1\}^{s}, g_{1}, \ldots, g_{k}$ and $h_{1}, \ldots, h_{k}$ over $\{0,1\}^{s}$ such that

$$
f=H \circ F_{1} \circ G
$$

where

$$
\begin{gathered}
G: \quad\left(\{0,1\}^{s}\right)^{k} \rightarrow\left(\{0,1\}^{s}\right)^{k} \\
\left(x_{1}, \ldots, x_{k}\right) \rightarrow\left(g_{1}\left(x_{1}\right), \ldots, g_{k}\left(x_{k}\right)\right)
\end{gathered}
$$

and

$$
\begin{aligned}
& H: \quad\left(\{0,1\}^{s}\right)^{k} \rightarrow\left(\{0,1\}^{s}\right)^{k} \\
& \left(x_{1}, \ldots, x_{k}\right) \rightarrow\left(h_{1}\left(x_{1}\right), \ldots, h_{k}\left(x_{k}\right)\right)
\end{aligned}
$$

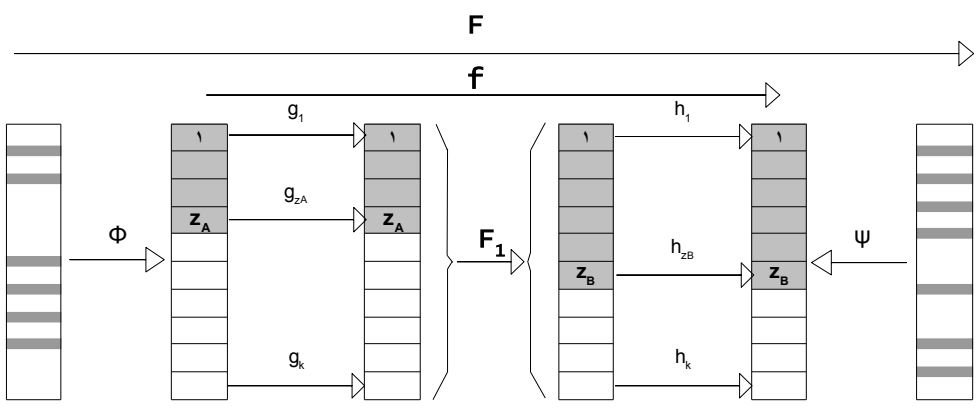

Fig. 5. Representation of $F$ from Problem 2 


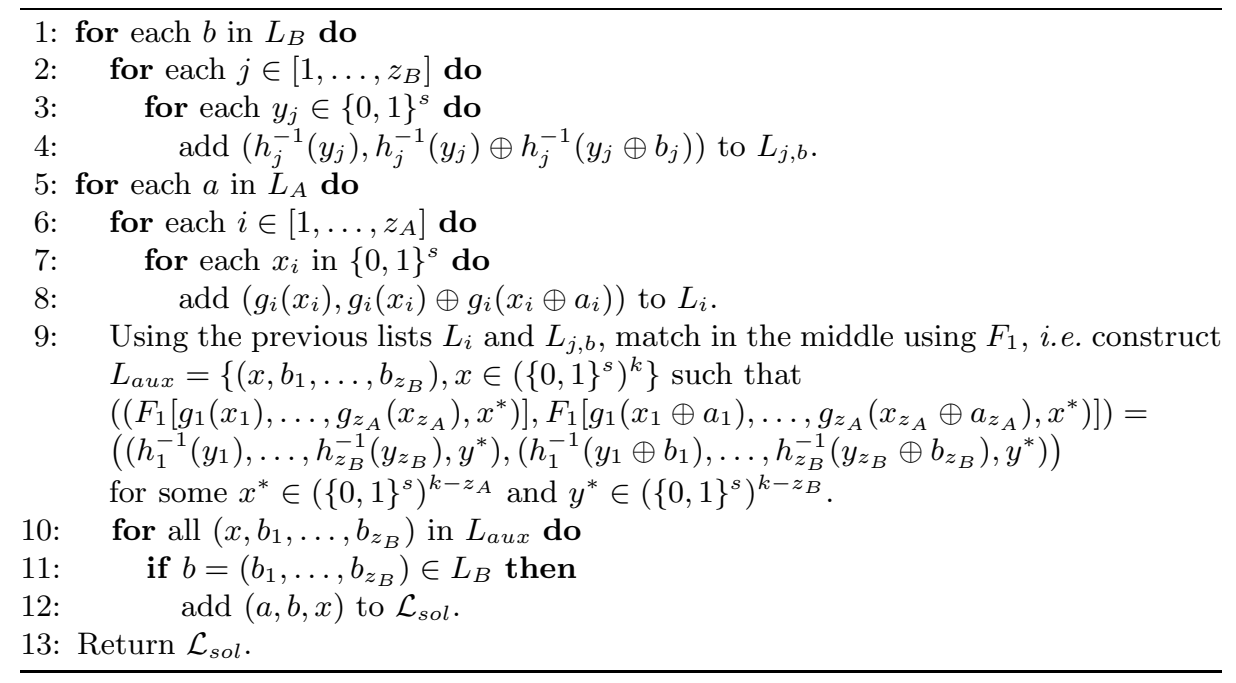

Fig. 6. General scheme of stop-in-the-middle algorithms

It is worth noting that we assume that both decompossitions $\phi$ and $\psi$ have been chosen in an appropriate way such that the $z_{A}$ words of $a$ (respectively the $z_{B}$ words of $b$ ) correspond to the first words of the input state (respectively of the output state). We call stop-in-the-middle algorithms those that solve Problem 2 following the main general scheme described in Fig. 6. The associated complexities depend on the particular form of $F_{1}$, as we show in the next sections.

In the cases we have studied and that we detail below, the function $f$ is formed by several AES transformations in parallel. We then expect $2^{l_{A}+l_{B}}$ solutions, as for each $a \in L_{A}$ and each $b \in L_{B}$ there exists one $c \in L_{C}$ so that the condition of Problem 2 holds. The match-in-the-middle step is assumed to be simple due to the simple form of $F_{1}$ (typical functions $F_{1}$ are linear diffusion layers). For the same reason, $L_{a u x}$ can typically be written in a compact way, for example, in several independent lists.

\subsection{Algorithm for LANE-256}

Each lane of the internal state of LANE-256 is composed of two AES states. An AES state is a state of size 128 bits that can be seen as a $4 \times 4$ matrix of bytes. The AES transformations are noted: SB for SubBytes, SR for ShiftRows and MC for MixColumn. The transformation SC mixes the two AES states at the end of each round by interchanging their columns. We consider Fig. 7 that represents a part of the differential path used in 12. In that attack it was treated as the merging of two inbounds and $2^{64}$ solutions were found with a complexity of $2^{96}$ in time and $2^{88}$ in memory. We consider the scheme represented in Fig. 7 where we have swapped lines and columns for a more easy intuitive understanding (so $\mathrm{SR}$ is applied to the columns and MC is applied to the lines). 


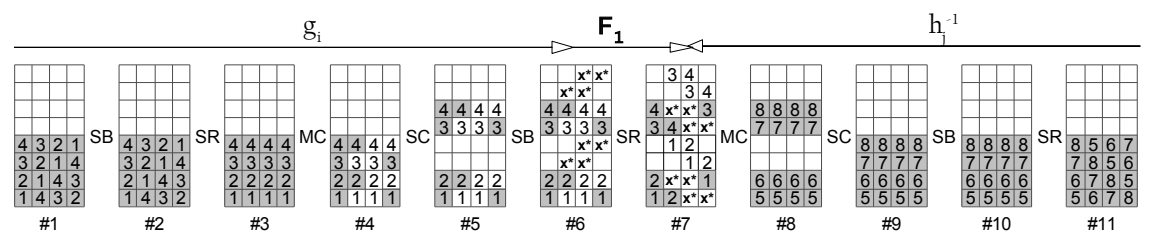

Fig. 7. Differential path associated to the first improvement on the LANE analysis

Using the example from $\left[12, l_{A}=32\right.$ and $l_{B}=32$ and $L_{C}$ is the list of all possible input values and needs to be neither stored nor computed. We consider that the input state (respectively the output state) of the function $f$ presented in Fig. 7 is decomposed into eight 32-bit words (i.e. $s=32$ and $k=8$ ). The input differences and output differences that we consider in $L_{A}$ and $L_{B}$ correspond to the first $z_{A}=z_{B}=432$-bit words of the state. In Fig. [7] each one of the $4+4=832$-bits active word corresponds to the four active bytes with the same number written on them ( 1 to 4 for the four active input words and 5 to 8 for the 4 active output words).

With the algorithm described in Fig. 8 we find the $2^{64}$ solutions with a complexity of $2^{66}$ in time and $2^{65}$ in memory. The time complexity associated to the studied path is $z_{B} 2^{l_{B}+32}+2^{l_{A}+32}$. This comes from the fact that each $L_{i}$ has average size $2^{16}$. Then, $L_{5,6}$ and $L_{7,8}$ have size $2^{l_{B}+32}$. Then the size of both $L_{\text {aux }}^{0}$ and $L_{\text {aux }}^{1}$ is $2^{l_{B}}$ since in each we keep the pairs of elements that match on 4 active bytes, and this happens with a probability of $2^{-64}$ (32 values and 32 differences); and the number of possible pairs is $2^{16+16+l_{B}+32}$. The memory complexity is $2^{l_{B}+32+1}+2^{32+1}+2^{l_{A}+l_{B}}$ for obtaining $2^{l_{A}+l_{B}}$ solutions. In [15] a detailed explanation on how this algorithm allows to considerably reduce the complexity of the LANE-256 semi-free-start collision presented in [12] is given, when applied jointly with other improvements concerning other steps of the attack.

\subsection{Algorithm for ECHO-256}

An ECHO-256 state is a state of size 2048 bits that can be seen as a $4 \times 4$ matrix of AES states. The ECHO operations BigSR, BigMC and BigSB are similar to the AES ones, but they operate on AES states instead of bytes. A SuperSbox is an Sbox defined by $\mathrm{SR} \circ \mathrm{SB} \circ \mathrm{MC} \circ \mathrm{SR} \circ \mathrm{SB}$. Applied on an AES state, it can be seen as a $32 \times 32$ Sbox. We define a SuperSbox set as each one of the 4 (in the AES state) sets of bits that act as input and output of the SuperSbox. We define a BigSuperSbox as an Sbox defined by BigSR ० BigSB ० BigMC $\circ$ BigSR $\circ$ BigSB. Applied to ECHO it defines 4 sets of size 4 AES-states.

We consider Fig. 9] where each column represents the four AES states that form a BigSuperSbox at a certain state \#i, for $i$ from 1 to 13. Each possible differences in $\# 1$ in $L_{A}$ consist of $z_{A}=12$ 32-bit words and the possible 
Require: Function $f$ and lists $L_{A}$ and $L_{B}$ of differences in $\# 1$ and \#11 respectively.

Ensure: List $\mathcal{L}_{\text {sol }}=\left\{(a, b, c)\right.$ such that $\left.f\left(c \oplus\left(a, 0^{s\left(k-z_{A}\right)}\right)\right) \oplus f(c)=\left(b, 0^{s\left(k-z_{B}\right)}\right)\right\}$.

1: for each $b$ in $L_{B}$ do

2: $\quad$ for $i$ from 5 to 8 do

3: $\quad$ for each $y \in\{0,1\}^{32}$ do

4: $\quad$ if $h_{i}^{-1}(y) \oplus h_{i}^{-1}\left(y \oplus b_{i}\right)$ has only the two bytes active (see state \#' $)$ then

5: $\quad$ Store $\left(y, b_{i}, h_{i}^{-1}(y), h_{i}^{-1}\left(y \oplus b_{i}\right)\right)$ in $L_{i}$, where the last two terms are truncated to the 2 active bytes.

6: $\quad$ for each $\left(y_{5}, b_{5}, u_{5}, w_{5}\right)$ from $L_{5}$ and $\left(y_{6}, b_{6}, u_{6}, w_{6}\right)$ from $L_{6}$ do

7: $\quad$ Add $\left(u_{5}, w_{5}, u_{6}, w_{6}, y_{5}, y_{6}, b_{5}, b_{6}\right)$ in $L_{5,6}$ indexed by $u_{5}, w_{5}, u_{6}, w_{6}$.

8: $\quad$ for each $\left(y_{7}, b_{7}, u_{7}, w_{7}\right)$ from $L_{7}$ and $\left(y_{8}, b_{8}, u_{8}, w_{8}\right)$ from $L_{8}$ do

9: $\quad$ Add $\left(u_{7}, w_{7}, u_{8}, w_{8}, y_{7}, y_{8}, b_{7}, b_{8}\right)$ in $L_{7,8}$ indexed by $u_{7}, w_{7}, u_{8}, w_{8}$.

10: $\quad$ Empty $L_{5}, L_{6}, L_{7}$ and $L_{8}$.

11: for each $a$ in $L_{A}$ do

12: for $i$ from 1 to 4 do

13: for each $x_{i} \in\{0,1\}^{32}$ do

14: $\quad$ if $g_{i}\left(x_{i}\right) \oplus g_{i}\left(x_{i} \oplus a_{i}\right)$ has only the two bytes active (see state \#4) then

15: $\quad$ Store $\left(x_{i}, g_{i}\left(x_{i}\right), g_{i}\left(x_{i} \oplus a_{i}\right)\right)$ in $L_{i}$, where the two last terms are truncated to the 2 active bytes.

16: for $i$ from 0 to 1 do

17: $\quad$ for each $\left(x_{2 i+1}, u_{2 i+1}, w_{2 i+1}\right)$ in $L_{2 i+1}$ and $\left(x_{2 i+2}, u_{2 i+2}, w_{2 i+2}\right)$ in $L_{2 i+2}$ do

18: if there exists an element in $L_{5+2 i, 6+2 i}$ indexed by $\left(u_{2 i+1}, w_{2 i+1}, u_{2 i+2}, w_{2 i+2}\right)$ then

19: $\quad$ Add $\left(x_{2 i+1}, x_{2 i+2}, b_{5+2 i}, b_{6+2 i}\right)$ to $L_{a u x}^{i}$ indexed by $\left(b_{5+2 i}, b_{6+2 i}\right)$.

20: $\quad$ for each $\left(x_{1}, x_{2}, b_{5}, b_{6}\right)$ in $L_{a u x}^{0}$ do

21: $\quad$ for each $\left(b_{7}, b_{8}\right)$ such that $\left(b_{5}, b_{6}, b_{7}, b_{8}\right) \in L_{B}$ do

22: $\quad$ if there exists an element in $L_{\text {aux }}^{1}$ indexed by $\left(b_{7}, b_{8}\right)$ then

23: $\quad$ add $\left(a,\left(b_{5}, b_{6}, b_{7}, b_{8}\right),\left(x_{1}, x_{2}, x_{3}, x_{4}\right)\right)$ to $\mathcal{L}_{\text {sol }}$.

24: Return $\mathcal{L}_{\text {sol }}$.

Fig. 8. Algorithm for solving two inbounds of LANE-256

differences in \#13 consist of $z_{B}=832$-bit words, where $L_{B}$ can be written as $L_{B}=L_{B_{1}} \times L_{B_{2}}$ with both $L_{B_{1}}$ (associated to AES state $B_{1}$ in Fig. 9) and $L_{B_{2}}$ (associated to AES state $\left.B_{4}\right)$ are subsets of $\left(\{0,1\}^{32}\right)^{4}$ each of size $2^{32}$ (this is a particular case which has to be adapted in other cases). Finding solutions for this differential path with the previously mentioned conditions is a problem proposed in [18] and was solved in such a way that $2^{32}$ solutions could be found with a complexity of $2^{128}$ in time and $2^{37}$ in memory. We propose here a new algorithm that can solve it for obtaining $2^{64}$ solutions with the same time complexity and a memory of $2^{67}$. Variants of our algorithm can be applied in several cases, like when the transition in $\# 7$ to $\# 8$ is from 2 active states to 3 , or from 1 to 4 or from 4 to 1 . Additionally we believe that it can improve the complexity of other future attacks on ECHO-256.

The list $L_{C}$ contains all the possible values for the input state. This list needs to be neither computed nor stored. Here the aim is to find for each possible $\left(a, b^{1}, b^{2}\right)$ in $L_{A} \times L_{B_{1}} \times L_{B_{2}}$ the associated $c$ so that $f(c) \oplus f\left(c \oplus\left(a, 0^{s\left(k-z_{A}\right)}\right)=\right.$ 


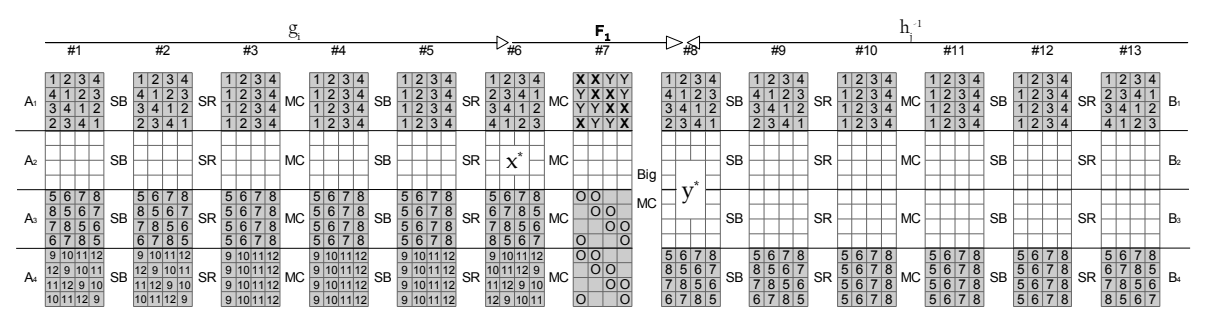

Fig. 9. Differential path on a BigSuperSbox of ECHO-256

$\left(b^{1}, b^{2}, 0^{s\left(k-z_{B}\right)}\right)$. In Fig. 9 we can see how the function $f$ can be written in the way requested by Problem 2. We omit the operation BigSR as it does not affect the states, as well as the round keys that are taken into account in the different $g_{i}$ and $h_{j}$. For the sake of simplicity we consider in Fig. 9 the list $L_{B}$ of possible differences before the last MC of the BigSuperSbox. This can be done by a simple transformation $\mathrm{MC}^{-1}$ of the differences in \#B'. The grey bytes represent the bytes with differences. We can observe that, from \#1 to \#6 there are $z_{A}=12$ independent active SuperSbox sets $(s=32)$, denoted in Fig. 9 by a number from 1 to 12 . To each of these groups we can associate a difference from $L_{A}$ and a value from $L_{C}$ at state \#1 and we can apply independently $g_{i}$, $i \in[1, \ldots, 12]$ to obtain the value and the difference of the group in \#6. The same way, from $\# 8$ to $\# 13$ there are $z_{B}=8$ independent active SuperSbox sets and the corresponding functions $h_{i}^{-1}, i \in[1, \ldots 8]$ that link state \#13 with state \#8. The function $F_{1}=\mathrm{MCoBigMC}$ takes a complete internal state in \#6 and computes the corresponding state in \#8. Let $f(x)=y$, and let $d_{i}^{\# 7}$ the $i$ th active diagonal in state \#7. Without knowing the values of $x^{*}$ nor of $y^{*}$ represented in Fig. 9 we can still write the following equations that have to be verified, that are obtained from BigMC, and that are used in the algorithm:

$$
2 \times d_{i}^{\# 7} \oplus d_{i+4}^{\# 7} \oplus d_{i+8}^{\# 7} \oplus 9 \times d_{i}^{\# 7} \oplus 3 \times d_{i+4}^{\# 7} \oplus 6 \times d_{i+8}^{\# 7}=h_{i}^{-1}\left(y_{i}\right) \oplus 3 \times h_{i+4}^{-1}\left(y_{i+4}\right)
$$

for $i \in 1, \ldots, 4$ where the multiplication corresponds to the one in the definition of MC applied independently to each byte of the diagonal.

We consider that the input state (respectively the output state) of the function presented in Fig. 9 is decomposed into sixteen 32-bit words (i.e. $s=32$ and $k=16)$. The input differences (respectively output differences) that we consider in $L_{A}\left(L_{B}\right)$ correspond to the first $z_{A}=12\left(z_{B}=8\right) 32$-bit words of the state. In Fig. 9 each one of the 12 (respectively 8) 32-bits active word from the input (respectively the output) corresponds to the four active bytes with the same number written on them ( 1 to 12 for the twelve active input words and 1 to 8 for the eight active output words).

Let $V_{X}\left(V_{Y}, V_{O}\right.$ respectively) be the values at the positions in \#7 marked with an $X(Y, O)$ and $\Delta_{X}\left(\Delta_{Y}, \Delta_{O}\right)$ their differences. Let $\Delta_{j^{\prime}}^{\# r}$ be an auxiliary variable denoting the difference for the SuperSbox set $j^{\prime}$ in state $\# r$. The algorithm is described in Fig. 10. 
Require: Function $f$, lists of differences $L_{A}$ (in \#1) and $L_{B_{1}}$ and $L_{B_{2}}$ (in \#13).

Ensure: $\mathcal{L}_{\text {sol }}=\left\{\left(a, b^{1}, b^{2}, c\right)\right.$, such that $\left.f(c) \oplus f\left(c \oplus\left(a, 0^{s\left(k-z_{A}\right)}\right)\right)=\left(b^{1}, b^{2}, 0^{s\left(k-z_{B}\right)}\right)\right\}$

\section{1: for $j$ from 1 to 4 do}

2: for each $y_{j} \in\{0,1\}^{32}$ and for each $b^{1}$ from $L_{B_{1}}$ do

3: $\quad$ Store $\left(h_{j}^{-1}\left(y_{j}\right), h_{j}^{-1}\left(y_{j}\right) \oplus h_{j}^{-1}\left(y_{j} \oplus b_{j}^{1}\right)\right)$ in $L_{\# 8, b^{1}}^{j}\left(4 \times 2^{32}\right.$ lists of size $\left.2^{32}\right)$.

4: for $j$ from 5 to 8 do

5: $\quad$ for each $y_{j} \in\{0,1\}^{32}$ and for each $b^{2}$ from $L_{B_{2}}$ do

6: $\quad$ Store $\left(h_{j}^{-1}\left(y_{j}\right), h_{j}^{-1}\left(y_{j}\right) \oplus h_{j}^{-1}\left(y_{j} \oplus b_{j}^{2}\right)\right)$ in $L_{\# 8, b^{2}}^{j}\left(4 \times 2^{32}\right.$ lists of size $\left.2^{32}\right)$.

7: for each $a$ in $L_{A}$ do

8: $\quad$ for $i$ from 1 to 12 do

9: $\quad$ for each $x_{i} \in\{0,1\}^{32}$ do

10: $\quad$ Store $\left(g_{i}\left(x_{i}\right), g_{i}\left(x_{i}\right) \oplus g_{i}\left(x_{i}, a_{i}\right)\right)$ in $L_{\# 6}^{i}$.

11: for $\Delta_{X}$ from 0 to $2^{64}-1$ (and not the 128 bits as done in [18]) do

12: $\quad$ Compute $\Delta_{O}$ and $\Delta_{j^{\prime}}^{\# 8}$ for $j^{\prime} \in\{1,2,5,6\}$ (with BigMC and linear condit.).

13: $\quad$ for each $b^{1}$ in $L_{B_{1}}$ and for $j=[1,2]$ do

14: $\quad$ Find an element in $L_{\# 8, b^{1}}^{j}$ such that $h_{j}^{-1}\left(y_{j}\right) \oplus h_{j}^{-1}\left(y_{j} \oplus b_{j}^{1}\right)=\Delta_{j}^{\# 8}$ and store $\left(h_{1}^{-1}\left(y_{1}\right), \Delta_{1}^{\# 8}, h_{2}^{-1}\left(y_{2}\right), \Delta_{2}^{\# 8}, b^{1}\right)$ in $L_{a u x_{1}}$.

15: $\quad$ for each $b^{2}$ in $L_{B_{2}}$ and for $j=[5,6]$ do

16: $\quad$ Find an element in $L_{\# 8, b^{2}}^{j}$ such that $h_{j}^{-1}\left(y_{j}\right) \oplus h_{j}^{-1}\left(y_{j} \oplus b_{j}^{2}\right)=\Delta_{j}^{\# 8}$ and store $\left(h_{5}^{-1}\left(y_{5}\right), \Delta_{5}^{\# 8}, h_{6}^{-1}\left(y_{6}\right), \Delta_{6}^{\# 8}, b^{2}\right)$ in $L_{a u x_{2}}$.

17: for each $\left(h_{1}^{-1}\left(y_{1}\right), \Delta_{1}^{\# 8}, h_{2}^{-1}\left(y_{2}\right), \Delta_{2}^{\# 8}, b^{1}\right)$ in $L_{a u x_{1}}$ and for each $\left(h_{5}^{-1}\left(y_{5}\right), \Delta_{5}^{\# 8}, h_{6}^{-1}\left(y_{6}\right), \Delta_{6}^{\# 8}, b^{2}\right)$ in $L_{a u x_{2}}$ do

18: $\quad$ Compute $V_{1}^{\prime}=h_{1}^{-1}\left(y_{1}\right) \oplus 3 \times h_{5}^{-1}\left(y_{5}\right)$ and $V_{2}^{\prime}=h_{2}^{-1}\left(y_{2}\right) \oplus 3 \times h_{6}^{-1}\left(y_{6}\right)$, and store $\quad\left(\left(h_{1}^{-1}\left(y_{1}\right), \Delta_{1}^{\# 8}, h_{2}^{-1}\left(y_{2}\right), \Delta_{2}^{\# 8}, b^{1}\right),\left(h_{5}^{-1}\left(y_{5}\right), \Delta_{5}^{\# 8}, h_{6}^{-1}\left(y_{6}\right), \Delta_{6}^{\# 8}, b^{2}\right)\right)$ in a hash table $T$ indexed by these $\left(V_{1}^{\prime}, V_{2}^{\prime}\right)$.

19: $\quad$ for $\Delta_{Y}$ from 0 to $2^{64}-1$ do

20: $\quad$ Determine by BigMC $\Delta_{j^{\prime}}^{\# 8}$ for $j^{\prime}=3,4,7,8$; and $\Delta_{j}^{\# 6}$ for $j \in[1, \ldots, 12]$.

21: $\quad$ for $i$ from 1 to 12 do

22 :

Find the element from $L_{\# 6}^{i}$ such that $g_{i}\left(x_{i}\right) \oplus g_{i}\left(x_{i}, a_{i}\right)=\Delta_{i}^{\# 6}$.

23: $\quad$ Compute with them by MC the values $d_{i}^{\# 7}$ of the active diagonals in \#7 and $V_{j}=2 \times d_{j}^{\# 7} \oplus d_{j+4}^{\# 7} \oplus d_{j+8}^{\# 7} \oplus 9 \times d_{j}^{\# 7} \oplus 3 \times d_{j+4}^{\# 7} \oplus 6 \times d_{j+8}^{\# 7}$ for $j=1,2$.

24: if there is an element such that $V_{1}^{\prime}=V_{1}$ and $V_{2}^{\prime}=V_{2}$ in $T$ (one on average, determines $b^{1}$ and $\left.b^{2}\right)$ then

25:

26:

27:

28:

Find $\left(h_{j^{\prime}}^{-1}\left(y_{j^{\prime}}\right), \Delta_{j^{\prime}}^{\# 8}\right)$ from $L_{\# 8, b^{1}}^{j^{\prime}}$ for $j^{\prime}=3,4$. This implies $y_{3}$ and $y_{4}$.

Find $\left(h_{j^{\prime}}^{-1}\left(y_{j^{\prime}}\right), \Delta_{j^{\prime}}^{\# 8}\right)$ from $L_{\# 8, b^{2}}^{j^{\prime}}$ for $j^{\prime}=7,8$. This implies $y_{7}$ and $y_{8}$.

if with these values of $\left(h_{j^{\prime}}^{-1}\left(y_{j^{\prime}}\right), j^{\prime}=3,4,7,8\right.$ and the ones obtained in step 22 of $g_{i}\left(x_{i}\right)$ for $i=3,4,7,8,11,12$, the equation (11) for $i=3,4$ derived from $F_{1}$ can be verified (happens with a probability of $2^{-64}$ ) then

29: Return $\mathcal{L}_{\text {sol }}$, containing about $2^{64+l_{A}}$ elements.

Fig. 10. Algorithm for finding solutions for one ECHO BigSuperSbox

So the time complexity is $\mathcal{O}\left(z_{B} 2^{l_{B_{1}}+s}+z_{B} 2^{l_{B_{2}}+s}+z_{A} 2^{s}+2^{l_{A}+64}\left(2^{l_{B_{1}}}+\right.\right.$ $\left.\left.2^{l_{B_{2}}}+2^{l_{B_{1}}} 2^{l_{B_{2}}}+z_{A} 2^{64}\right)\right)$. The memory complexity is $\mathcal{O}\left(z_{B} 2^{l_{B_{1}}+s}+z_{B} 2^{l_{B_{2}}+s}+\right.$

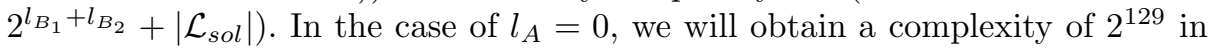


time and $2^{66}$ in memory for obtaining $2^{64}$ solutions. This algorithm proposes several trade-offs when changing the values of $\left|\Delta_{X}\right|$, and can be adapted for other forms of $L_{B}$.

\section{How to Improve the Best Known Attacks on Five SHA-3 Candidates}

In this section we enumerate briefly the main algorithms or ideas that we use to improve the best known attacks on the hash functions JH, Grøstl, ECHO, Luffa and LANE as shown on Table 1] In the full version of the paper [15] more detailed descriptions are provided.

- JH: To improve the complexities over the ones in 17 we use the instantmatching (as in Section 2.1) and gradual-matching algorithms as well as the fact that we do not merge the lists until we really have to (to keep lists of smaller sizes, with a smaller complexity).

- Grøstl: Instead of the initial lists used in [16, we can define them so that we erase the elements that for sure won't verify the outbound part. Having lists of smaller size translates to a smaller complexity.

- ECHO: Using conviniently the algorithm from Section 3.2 we provide better trade-offs improving the time complexity from [18.

- Luffa: The parallel-matching algorithm is applied in [10, improving the time complexity over the brute force merging method by increasing the memory requirements. If we apply instead the gradual-matching algorithm, the time complexity can still be better than the brute force one while the memory needs are not increased.

- LANE: In the cases of LANE-256 and LANE-512 several improvements are applied at different steps of the attacks from [12. They use the instantmatching algorithm, as well as some more appropriate ways to formulate the problem, and the algorithms from Section 3.1 and from [15, App.B].

\section{Conclusion}

The main contribution of this paper is to propose several algorithms for solving the problem which constitutes the bottleneck of most rebound attacks, leading to improvements of the previously known complexities. We also highlight the importance of identifying the situations that could help improving the complexity of this type of attacks. This is often a difficult task due to the high technicality of the attacks and algorithms.

Finally, the previous contributions lead to improvements of most of the best known rebound attacks applied to the SHA-3 candidates JH, Grøstl, Luffa, ECHO-256 and LANE. It is important to point out that we just tried to improve the complexities of existing attacks. However, the work presented in this paper can be very useful for future rebound attacks, in particular we believe that the attacks on $\mathrm{JH}$ and on the compression function of ECHO can be improved 
(extending the number of rounds attacked) by exploiting the algorithms and ideas presented here. Finally, we believe that some of these algorithms, specially those of Section 2, will be applicable in other contexts besides rebound attacks.

Acknowledgements. The author would like to thank Willi Meier, Simon Knellwolf, Marine Minier, Thomas Peyrin, Martin Schläffer, Joana Treger and Fabien Viger for many helpful comments and discussions. A special mention is needed for Anne Canteaut and Andrea Röck for all the help and suggestions to improve this paper.

\section{References}

1. Barreto, P.S.L.M., Rijmen, V.: The Whirlpool Hashing Function (revised in 2003)

2. Benadjila, R., Billet, O., Gilbert, H., Macario-Rat, G., Peyrin, T., Robshaw, M., Seurin, Y.: Sha-3 proposal: ECHO. Submission to NIST (2009) (updated)

3. Camion, P., Patarin, J.: The knapsack hash function proposed at crypto 1989 can be broken. In: Davies, D.W. (ed.) EUROCRYPT 1991. LNCS, vol. 547, pp. 39-53. Springer, Heidelberg (1991)

4. Canniere, C.D., Sato, H., Watanabe, D.: Hash Function Luffa: Specification. Submission to NIST (2009) (Round 2)

5. Canteaut, A., Naya-Plasencia, M.: Structural weaknesses of permutations with low differential uniformity and generalized crooked functions. In: Finite Fields: Theory and Applications - Selected Papers from the 9th International Conference Finite Fields ans Applications. Contemporary Mathematics, vol. 518, pp. 55-71. AMS, Providence (2010), http://www-rocq.inria.fr/secret/Maria.Naya_Plasencia/ papers/canteaut-nayaplasencia.pdf

6. Chose, P., Joux, A., Mitton, M.: Fast correlation attacks: An algorithmic point of view. In: Knudsen, L.R. (ed.) EUROCRYPT 2002. LNCS, vol. 2332, pp. 209-221. Springer, Heidelberg (2002)

7. Gauravaram, P., Knudsen, L.R., Matusiewicz, K., Mendel, F., Rechberger, C., Schläffer, M., Thomsen, S.S.: Grøstl - a SHA-3 candidate. Submitted to the SHA-3 competition, NIST (2008), http://www.groestl.info

8. Gilbert, H., Peyrin, T.: Super-sbox cryptanalysis: Improved attacks for AESlike permutations. In: Hong, S., Iwata, T. (eds.) FSE 2010. LNCS, vol. 6147, pp. 365-383. Springer, Heidelberg (2010)

9. Indesteege, S.: The Lane hash function. Submitted to the SHA-3 competition, NIST (2008), http://www.cosic.esat.kuleuven.be/publications/article-1181.pdf

10. Khovratovich, D., Naya-Plasencia, M., Röck, A., Schläffer, M.: Cryptanalysis of Luffa v2 Components. In: Biryukov, A., Gong, G., Stinson, D.R. (eds.) SAC 2010. LNCS, vol. 6544, pp. 388-409. Springer, Heidelberg (2011)

11. Lamberger, M., Mendel, F., Rechberger, C., Rijmen, V., Schläffer, M.: Rebound Distinguishers: Results on the Full Whirlpool Compression Function. In: Matsui, M. (ed.) ASIACRYPT 2009. LNCS, vol. 5912, pp. 126-143. Springer, Heidelberg (2009)

12. Matusiewicz, K., Naya-Plasencia, M., Nikolić, I., Sasaki, Y., Schläffer, M.: Rebound Attack on the Full Lane Compression Function. In: Matsui, M. (ed.) ASIACRYPT 2009. LNCS, vol. 5912, pp. 106-125. Springer, Heidelberg (2009) 
13. Mendel, F., Rechberger, C., Schläffer, M., Thomsen, S.S.: The Rebound Attack: Cryptanalysis of Reduced Whirlpool and Grøstl. In: Dunkelman, O. (ed.) FSE 2009. LNCS, vol. 5665, pp. 260-276. Springer, Heidelberg (2009)

14. Mendel, F., Peyrin, T., Rechberger, C., Schläffer, M.: Improved cryptanalysis of the reduced gr $\varnothing$ stl compression function, ECHO permutation and AES block cipher. In: Jacobson Jr., M.J., Rijmen, V., Safavi-Naini, R. (eds.) SAC 2009. LNCS, vol. 5867, pp. 16-35. Springer, Heidelberg (2009)

15. Naya-Plasencia, M.: How to Improve Rebound Attacks. Cryptology ePrint Archive, Report 2010/607 (2010), http://eprint.iacr.org/2010/607.pdf (extended version)

16. Peyrin, T.: Improved Differential Attacks for ECHO and Grøstl. In: Rabin, T. (ed.) CRYPTO 2010. LNCS, vol. 6223, pp. 370-392. Springer, Heidelberg (2010)

17. Rijmen, V., Toz, D., Varıcı, K.: Rebound Attack on Reduced-Round Versions of JH. In: Hong, S., Iwata, T. (eds.) FSE 2010. LNCS, vol. 6147, pp. 286-303. Springer, Heidelberg (2010)

18. Sasaki, Y., Li, Y., Wang, L., Sakiyama, K., Ohta, K.: Non-full-active Super-Sbox Analysis: Applications to ECHO and Grøstl. In: Abe, M. (ed.) ASIACRYPT 2010. LNCS, vol. 6477, pp. 38-55. Springer, Heidelberg (2010)

19. Schroeppel, R., Shamir, A.: A $\mathrm{T}=\mathrm{O}\left(2^{\mathrm{n} / 2}\right), \mathrm{S}=\mathrm{O}\left(2^{\mathrm{n} / 4}\right)$ algorithm for certain NPcomplete problems. SIAM J. Comput. 10(3), 456-464 (1981)

20. Wagner, D.: A generalized birthday problem. In: Yung, M. (ed.) CRYPTO 2002. LNCS, vol. 2442, pp. 288-303. Springer, Heidelberg (2002)

21. Wu, H.: The hash function JH. Submission to NIST (2009) (updated), http://icsd.i2r.a-star.edu.sg/staff/hongjun/jh/jh_round2.pdf

22. Wu, S., Feng, D., Wu, W.: Cryptanalysis of the LANE hash function. In: Jacobson Jr., M.J., Rijmen, V., Safavi-Naini, R. (eds.) SAC 2009. LNCS, vol. 5867, pp. 126-140. Springer, Heidelberg (2009) 